\title{
Semantic transparency and masked morphological priming: The case of prefixed words
}

\author{
KeVin Diependaele \\ Ghent University, Ghent, Belgium \\ DOMINIEK SANDRA \\ University of Antwerp, Antwerp, Belgium \\ AND \\ JONATHAN GRAINGER \\ CNRS \& Aix-Marseille University, Aix-Marseille, France
}

\begin{abstract}
In four lexical decision experiments, we investigated masked morphological priming with Dutch prefixed words. Reliable effects of morphological relatedness were obtained with visual primes and visual targets in the absence of effects due to pure form overlap. In certain conditions, priming effects were significantly greater with semantically transparent prefixed primes (e.g., rename-name) relative to the priming effects obtained with semantically opaque prefixed words (e.g., relate-late), even with very brief ( 40 -msec) prime durations. With visual primes and auditory targets (cross-modal priming), significant facilitation was found in all related prime conditions, independent of whether or not primes were morphologically related to targets. The results are interpreted within a bimodal hierarchical model of word recognition in which morphological effects arise through the interplay of sublexical (morpho-orthographic) and supralexical (morpho-semantic) representations. The word stimuli from this study may be downloaded as supplemental materials from http://mc.psychonomic-journals .org/content/supplemental.
\end{abstract}

Psycholinguistic experiments commonly show that a word is recognized faster following a morphologically related prime word than following an unrelated prime (e.g., viewer-view vs. sooner-view; see, e.g., Marslen-Wilson, Tyler, Waksler, \& Older, 1994). Recognition times are usually measured as reaction times (RTs) in the wordnonword (lexical) decision task. Importantly, priming from morphological relatives is not only evident in comparison with unrelated prime-target pairs, but also with matched form-related and meaning-related items (e.g., viewer-view vs. freeze-free and bus-car). Furthermore, this pattern is also obtained in the visual masked priming paradigm (Forster \& Davis, 1984), in which primes are unavailable for conscious report because of masking and a very brief presentation (e.g., Grainger, Colé, \& Segui, 1991). These morphological priming effects are typically interpreted as reflecting access to some form of explicit representation of morphological structure during the recognition of morphologically complex words (see Diependaele, Grainger, \& Sandra, in press, for a review).

As summarized in Diependaele, Grainger, and Sandra (in press), different mechanisms and architectures have been proposed to account for morphological influences on word recognition. One key theoretical difference that has generated much research in the last decade is whether morphological structure is represented at the level of form representations (i.e., in terms of morpho-orthographic or -phonological representations) and accessed prior to or simultaneous with whole-word (lexical) form representations (e.g., Schreuder \& Baayen, 1995; Taft, 1994; Taft $\&$ Forster, 1975) or, alternatively, at the level of amodal morpho-semantic representations accessed via wholeword form representations (e.g., Giraudo \& Grainger, 2000; Marslen-Wilson et al., 1994).

With respect to a possible role for morpho-orthographic representations, one key finding is that visual masked morphological priming with complex primes and stem targets not only occurs with semantically transparent primes (e.g., viewer-view), but also with semantically opaque and pseudocomplex primes (e.g., department-depart, corner-corn; e.g., Gold \& Rastle, 2007; Lavric, Clapp, \& Rastle, 2007; Longtin, Segui, \& Hallé, 2003; McCormick, Rastle, \& Davis, 2008; Rastle, Davis, Marslen-Wilson, \& Tyler, 2000; Rastle, Davis, \& New, 2004). The difference between opaque and pseudocomplex items is that, in the former case, the primes and targets are etymologically re- 
lated. Furthermore, nonword primes formed by an illegal combination of an existing stem and an existing suffix (e.g., quickify-quick) have been found to be just as effective as true suffixed primes (Longtin \& Meunier, 2005). These data show that morphological representations can be activated purely on the basis of sublexical orthographic characteristics; specifically, they imply a mechanism that groups letter sequences into morpheme-sized chunks. Morphological priming occurs whenever visual primes are fully composed of morphemic sequences (as is the case in corner, but not in freeze).

With respect to a possible role for morpho-semantic representations, the current evidence is not clear cut. The key finding would be a significant advantage for semantically transparent complex primes relative to both opaque and pseudocomplex primes in masked morphological priming (i.e., a semantic transparency effect). In a recent review, Rastle and Davis (2008) concluded that no such advantage exists. However, looking more closely at the evidence, it appears that studies almost always show a numerical advantage for transparent items (see Rastle \& Davis, 2008, Table 1) and that in some cases this advantage is supported statistically (e.g., Diependaele, Sandra, \& Grainger, 2005; Morris, Frank, Grainger, \& Holcomb, 2007). On the basis of these observations, we consider a hybrid account of morphological processing, including a role for both morpho-orthographic and morpho-semantic representations in the earliest stages of word recognition (Diependaele et al., 2005). This hybrid model is depicted in Figure 1.

According to the model in Figure 1, the internal morphological structure of printed words is represented at two distinct levels: (1) in terms of frequency-dependent clustering at the level of sublexical orthographic representations and (2) in terms of regularities in the mapping of word forms onto semantics. Rather than considering the representations at either level as morphological representations per se, they are better viewed as morphologically constrained orthographic and semantic representations (see Hay \& Baayen, 2005; Plaut \& Gonnerman, 2000; and Seidenberg \& Gonnerman, 2000, for a similar approach). When a letter sequence is processed, the visual input is mapped in parallel onto the morpho-orthographic and morpho-semantic representations (via whole-word form representations in the latter case). In a priming context, opaque morphological relatives will only be able to prime each other through shared representations at the morphoorthographic level, whereas transparent items will also be able to do this via shared representations at the morphosemantic level. Feedback connections in this hybrid model allow an online interplay between the two levels of morphological representation. Morpho-orthographic and morphosemantic activations will only be able to "resonate" in the case of semantically transparent complex words, which implies that morphological priming will gradually disappear in the case of opaque relatives. Evidence for morphoorthographic activation can therefore only be found with sufficiently short prime exposure durations (e.g., Longtin et al., 2003; Rastle et al., 2000). Finally, the feedback from morpho-semantic representations can also help the off-
Morpho-semantic

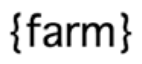

$\{\mathrm{er}\}$

Lexical form
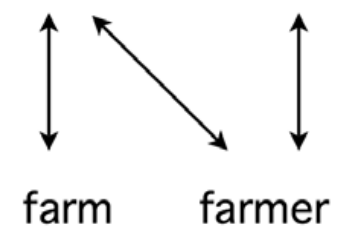

Morpho-orthographic
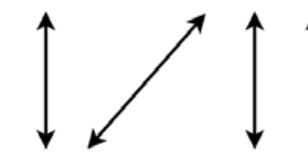

(farm)

(er)
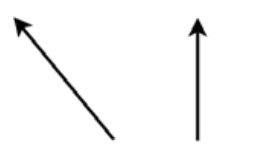

FARMER

Figure 1. The hybrid model of morphological processing, depicted from the perspective of a hierarchical interactive-activation account of word recognition. The input is mapped in parallel onto morpho-orthographic and morpho-semantic representations, via whole-word form representations in the latter case. Both online and offline interactions between morpho-orthographic and morpho-semantic representations are possible through feedback connections.

line development of morpho-orthographic representations during the process of learning to read (see Rastle \& Davis, 2008, for a similar proposal).

The goal of the present study is to further examine the plausibility of the hybrid account described above by investigating masked morphological priming with prefixed instead of suffixed items. A number of studies have reported differences between prefixed and suffixed words (e.g., Beauvillain, 1996; Colé, Beauvillain, \& Segui, 1989; Meunier \& Segui, 2002), which may reflect a beginningto-end sequential bias in the processing of printed words (e.g., Bergman, Hudson, \& Eling, 1988; Giraudo \& Grainger, 2003; Libben, 1994) and/or differences in distributional properties (e.g., Chateau, Knudsen, \& Jared, 2002; Hyman, 2008; Laudanna \& Burani, 1995; Wurm, 1997). Following a number of earlier masked priming studies with prefixed or suffixed items, however, there are no direct reasons to expect different morphological priming effects with prefixed words (Duñabeitia, Perea, \& Carreiras, 2008; Grainger et al., 1991). To our knowledge (see also Rastle \& Davis, 2008), the present study nevertheless remains the first to evaluate stem priming with both semantically transparent and opaque prefixed primes (e.g., rename-name and relate-late) and matched formcontrol primes (e.g., entail-tail) using brief prime exposure durations. ${ }^{1}$ Any observed differences with respect to prior masked priming evidence from suffixed words will inform future attempts to provide a comprehensive account of the processing of morphologically complex 
words during reading. Our study is primarily concerned with the conclusions that can be drawn regarding the early effects of semantic transparency for prefixed words.

The main empirical question here is whether it is possible to observe significantly stronger stem priming with semantically transparent prefixed primes (e.g., renamename) than with semantically opaque prefixed primes (e.g., relate-late) using brief prime exposure durations in the lexical decision task. If so, this would be consistent with rapid activation of morpho-semantic representations during visual word recognition, as postulated by the hybrid account. However, the presence of semantic transparency effects alone does not provide unequivocal support for the hybrid account. We also need to examine the relationship with morphological priming in the case of opaque items. If stem priming were significantly greater with opaque prefixed primes (e.g., relate-late) than with form-control primes (entail-tail), this would provide evidence for morpho-orthographic activation. Since the hybrid account predicts that inputs are mapped in parallel onto morpho-orthographic and morpho-semantic representations, a key prediction is that morphological priming for opaque items and semantic transparency effects should co-occur with sufficiently short prime durations. Alternative accounts predict that semantic transparency effects reflect the integration of morpho-orthographic activations during semantic processing, which is only successful for transparent items (e.g., Longtin et al., 2003; Schreuder \& Baayen, 1995). According to this proposal, evidence for morpho-orthographic activation should disappear quickly when transparency effects emerge in the data. All of the present experiments were conducted with Dutch materials and native Dutch-speaking participants. Table 1 gives a summary of the conditions tested in this study.

Finally, Experiments 1 and 2 not only tested visual masked priming, but also masked cross-modal priming with visual primes and auditory targets (Grainger, Diependaele, Spinelli, Ferrand, \& Farioli, 2003; Kouider \& Dupoux, 2001). Our prior research (Diependaele et al., 2005) revealed that cross-modal transfer is effective with respect to morpho-orthographic activation in the case of suffixed words. The data showed that both semantically transparent and opaque suffixed primes facilitated lexical decisions for auditory stem targets (e.g., viewer-view and corner-corn). In contrast, matched form controls (e.g., freeze-free) produced an inhibitory cross-modal effect. If the model shown in Figure 1 is extended according to the architecture of the bimodal interactive-activation model (e.g., Diependaele, Ziegler, \& Grainger, in press; Grainger

Table 1 Stimulus Types Used in All Experiments

\begin{tabular}{cccc}
\hline & Transparent & Opaque & Form \\
\hline Prefixed Words & & & \\
Dutch example & gegil & gebed & barok \\
English example & rename & relate & entail \\
Stems & & & \\
Dutch example & gil & bed & rok \\
English example & name & late & tail \\
\hline
\end{tabular}

\& Ferrand, 1994), transfer of morpho-orthographic activation could arise at the sublexical and/or the lexical level. At the sublexical level, the orthographic representation of view, activated through the morpho-orthographic decomposition of the prime viewer, would map onto the corresponding sublexical phonological representation. An alternative here is to assume that morphological decomposition occurs within the mapping of letters onto phonemes (see Diependaele et al., 2005). At the lexical level, the morpho-orthographic decomposition of the prime viewer would lead to the activation of the whole-word orthographic representation view, which would map onto the corresponding whole-word phonological representation.

Investigating prefixed instead of suffixed words allows us to further investigate cross-modal interactions in morphological processing. Research on auditory word recognition has highlighted a fundamental difference between priming effects with (1) word-initially overlapping primes and targets (e.g., carton-carrot) and (2) wordfinally overlapping primes and targets (e.g., fable-cable). Word-initial overlap produces inhibitory priming relative to unrelated primes, whereas word-final overlap produces facilitatory priming effects (e.g., Slowiaczek \& Hamburger, 1992; Slowiaczek, McQueen, Soltano, \& Lynch, 2000). Furthermore, the word-initial inhibitory priming effect only arises when the primes are words, whereas word-final facilitation arises independently of the prime's lexical status, indicating a lexical origin in the former case and a sublexical origin in the latter (Slowiaczek \& Hamburger, 1992). The inhibitory cross-modal priming with form-control items in Diependaele et al. (2005) appears to be consistent with these findings. In the present experiments, all of the related primes and targets have word-final instead of word-initial overlap. Hence, we expect to find facilitatory cross-modal form-priming effects, depending on the contribution of sublexical processing to the priming effects. Given the complex activation dynamics in a bimodal framework, such a shift in cross-modal form priming could give rise to a different morphological priming pattern than has been previously observed with suffixed items.

\section{EXPERIMENT 1}

\section{Method}

Participants. Forty undergraduate students of the University of Antwerp participated in Experiment 1. All were native speakers of Dutch and had normal or corrected-to-normal vision and no hearing impairment.

Materials and Design. The materials and design are discussed separately for the word and the nonword stimuli.

Word items. We selected 144 word pairs from the CELEX Dutch lexical database (Baayen, Piepenbrock, \& Gulikers, 1995) to serve as related primes and targets in the experiment (see the supplemental materials). Each pair contained a "longer" and a "shorter" word that shared (1) a transparent prefix-derived morphological relationship (e.g., gegil-gil "screaming-scream"; $n=48$ ), (2) an opaque prefixderived morphological relationship (e.g., gebed-bed "prayer-bed"; $n=48$ ), or (3) a mere form relationship (e.g., barok-rok "baroqueskirt"; $n=48$ ), defining the levels of the priming-type factor in the design. The shorter member of a pair never contained derivational affixes and always showed full orthographic and phonological (in- 
cluding syllabic) overlap with the ending of the longer word. We considered morphological transparency from a synchronic (as opposed to a diachronic) perspective. As a consequence, the opaque condition comprised both pairs with an etymological relationship and pairs with a mere structural morphological relationship (see also Rastle et al., 2004). In form pairs, the residual letter/phoneme sequence at the beginning of the longer word (i.e., ba-in barok-rok) never formed a possible Dutch prefix. In the transparent and opaque pairs, this sequence was never a prepositional prefix (e.g., $n a$ - in $n a$ zicht "revision"), since those morphemes also occur as freestanding prepositions (e.g., na "after"). Each prefix occurred equally often in the transparent and opaque pairs. Across the three conditions, pairs were matched as closely as possible on word length, surface frequency, and proportion of orthographic and phonological overlap (see Table 2). Finally, we asked 109 native speakers of Dutch to rate the semantic relatedness of each word pair on a seven-point scale $(0=$ highly unrelated, $6=$ highly related $)$. The respondents were contacted via an e-mail survey. Before we accepted the data of a participant, we made sure that he or she was a native speaker of Dutch and naive regarding the present research purposes. In line with our intuitions, Mann-Whitney $U$ tests on the median scores (see Table 2) were significantly higher for transparent pairs than for either opaque or form pairs (both $p \mathrm{~s}<.001$ ). Importantly, there was no significant difference between the scores for opaque and form pairs.

The shorter word in each pair served as a target, and the longer word served as its related prime. Unrelated primes were newly selected words that matched the related prime in letter length and frequency but that had no clear formal or semantic relation with the target. Using a Latin square design, we created four lists with word items by rotating relatedness (related vs. unrelated) and target modality (visual vs. auditory) within the levels of priming type (transparent vs. opaque vs. form). These lists were distributed uniformly across the participants.

Nonword items. The nonword primes and targets were generated from a new set of word pairs, matched as closely as possible to the critical word stimuli. We selected 144 new word pairs, equally divided into three subsets according to the levels of priming type. Unlike the word item subsets, each subset now contained 24 related pairs and 24 unrelated pairs (instead of 48 related pairs). The related pairs were selected following the same criteria used for the word items, and the unrelated pairs consisted of words without a clear formal or semantic similarity. In both cases, we made sure that the members of a pair respected the length and frequency range of the corresponding members in the word items. The shorter word in each pair served as a target, and the longer word served as the related or unrelated prime for the target. We then replaced a single letter in each target to transform it into a nonword. We made sure that the resulting nonword targets were al- ways regularly pronounceable in Dutch. We did not transform primes into nonwords so that the primes would not be informative about the lexical status of the targets. Because half of the initially selected word pairs were related and the other half were unrelated, there was no within-items manipulation of relatedness for the nonword items. Hence, only two lists of nonword items were created, by rotating target modality within the levels of relatedness and priming type. These lists were also distributed uniformly across the participants.

Procedure. The experimental session started with 24 practice trials. The procedure on these trials was identical to that in the experimental trials (see below). The stimuli were also similar (but not identical) to those in the experimental trials. The main experiment followed immediately after the practice session and consisted of 288 trials, presented without a break. Each participant encountered 72 visual word targets, 72 auditory word targets, 72 visual nonword targets, and 72 auditory nonword targets, presented in random order.

All visual stimuli appeared in a white fixed-width font (Courier New) against a black background on a monitor with a 75-Hz refresh rate (i.e., with a 13.33-msec refresh cycle). The font size for visual stimuli was set to 12-point, except for the visual targets, which were presented in 18-point. The auditory targets were recorded by a female native speaker of Dutch. During the experiment, they were presented via a Sennheiser HD 280 headset, connected to a standard PC soundboard. The experiment was run on a Windows XP operating system using the DMDX software package of Forster and Forster (2003).

On a given trial, the sequence of events was as follows. First, a forward mask (11 hash marks) appeared for $500 \mathrm{msec}$ at the center of the screen, together with two fixation marks: small vertical lines directly above and underneath the center of the mask. Immediately afterward, a lowercase prime appeared at the center of the screen for $67 \mathrm{msec}$ (i.e., five refresh cycles of the $75-\mathrm{Hz}$ video monitor). The prime was then replaced by the backward mask. This mask consisted of a pseudorandom sequence of 11 uppercase consonants. Prior to the experiment, we designed a separate backward mask for each target, taking care that the mask never contained any of the consonants in the target or in any of its associated primes. In the case of a visual target, an uppercase target replaced the backward mask after $13 \mathrm{msec}$ (i.e., one refresh cycle of the $75-\mathrm{Hz}$ video monitor). The target then remained on the screen until a response was recorded, or if no response followed, it was removed after 4,000 msec. Auditory targets were also presented $13 \mathrm{msec}$ after the backward mask appeared on the screen. However, the backward mask now remained on the screen and was removed when a response was recorded, or after 4,000 msec if no response followed. At the end of each trial (i.e., when a response was recorded or when the response deadline of 4,000 msec was exceeded), participants saw a blank (black) screen for $500 \mathrm{msec}$ before the next trial started.

Table 2

Stimulus Characteristics of the Related Primes and Targets

\begin{tabular}{|c|c|c|c|c|c|c|}
\hline & \multicolumn{2}{|c|}{ Transparent } & \multicolumn{2}{|c|}{ Opaque } & \multicolumn{2}{|c|}{ Form } \\
\hline & $M$ & $S D$ & $M$ & $S D$ & $M$ & $S D$ \\
\hline \multicolumn{7}{|l|}{ Prefixed Words } \\
\hline Letter length & 7.25 & 1.12 & 6.92 & 1.30 & 6.83 & 1.17 \\
\hline Phoneme length & 6.46 & 0.85 & 6.04 & 0.92 & 5.88 & 0.95 \\
\hline Surface frequency ${ }^{\mathrm{a}}$ & 16.25 & 64.42 & 18.62 & 23.62 & 18.30 & 38.04 \\
\hline \multicolumn{7}{|l|}{ Stems } \\
\hline Letter length & 5.00 & 1.11 & 4.67 & 1.14 & 4.56 & 0.99 \\
\hline Phoneme length & 4.23 & 0.83 & 3.83 & 0.72 & 3.77 & 0.72 \\
\hline Surface frequencya & 63.67 & 88.26 & 71.53 & 113.31 & 45.56 & 79.48 \\
\hline \multicolumn{7}{|l|}{ Prefixed Words vs. Stems } \\
\hline Letter overlap ${ }^{\mathrm{b}}$ & .69 & .08 & .67 & .08 & .67 & .08 \\
\hline Phoneme overlap ${ }^{\mathrm{b}}$ & .65 & .09 & .63 & .08 & .63 & .11 \\
\hline Semantic relatedness $(0-6)$ & 5.04 & 1.09 & 0.31 & 0.66 & 0.02 & 0.15 \\
\hline
\end{tabular}

aPer million. bProportion of position-specific form overlap with respect to the end of the word. 
Each participant sat approximately $50 \mathrm{~cm}$ from the computer screen in a dimly lit, soundproofed room. We asked the participants to prepare themselves for each trial by fixating the middle of the screen (i.e., the region between the two fixation marks). We told them that immediately after this preparation display, they would encounter one of four possible stimuli: (1) a Dutch word on the screen, (2) a "Dutch" pseudoword on the screen, (3) a Dutch word in the headphones, or (4) a "Dutch" pseudoword in the headphones. The task was explained as deciding as quickly and accurately as possible whether or not the perceived stimulus was a Dutch word. The participants were told to perform this task by pressing the corresponding right or left front button on a Logitec Wingman Precision USB game pad. Which of the two buttons a participant used for a word or for a nonword response during the experiment was left to his or her preference.

\section{Results}

Correct RTs and errors for word targets ${ }^{2}$ were analyzed using linear mixed-effects models with participants and items as (crossed) random variables (see Baayen, Davidson, \& Bates, 2008). There was no averaging of the data prior to the analyses. For the error data, we used generalized linear mixed-effects models with the logistic link function.

Since the variances of the RTs on intra- and cross-modal trials clearly differed, we analyzed the data separately for visual and auditory targets. We inverse-transformed all RTs (i.e., $-1 / \mathrm{RT}$ ) to reduce the positive skew in the distributions. Transformed RTs smaller than Q1 $-3 *$ IQR or larger than Q3 $+3 * \mathrm{IQR}$, by either participants or items, were excluded from the analyses (with Q1 = first quartile, Q3 = third quartile, and IQR = Q3 - Q1 = interquartile range). This trimming was done separately for visual and auditory targets and led to the removal of $0.4 \%$ of the data.

In each analysis, we looked at the interaction of relatedness (related vs. unrelated) with priming type (transparent vs. opaque vs. form). In the RT analyses, we also included a number of (procedural) control variables. Trial number was tested as a fixed effect with by-participants random slopes to control for (participant-specific) effects of fatigue and/or task familiarization. To control for the effects of (1) errors, (2) changes in the lexical status of targets, and (3) changes in the modality of targets, we looked at the effects of three additional factors - accuracy on the previous trial, lexical status of the previous target, and modality of the previous target - and their interactions.

Nonsignificant predictors were removed from the model (in a backward stepwise fashion). To reduce skew in the residuals of the final models, we refitted these models after removing observations with an absolute standardized residual greater than 3 . We obtained $p$ values via Markov chain Monte Carlo sampling (with a sample size of 10,000). For simplicity, we only report below the results regarding relatedness and priming type (see Table 3 for an overview). The results for the other predictors can be found in Appendix A.

Visual targets. The relatedness $\times$ priming type interaction was marginally significant in the RT analysis $[F(2,2821)=2.72, p=.06]$. There was positive priming for transparent and opaque items only $[t(2821)=3.66$,
Table 3

Condition Means and Priming Effects for Experiment 1

\begin{tabular}{|c|c|c|c|c|c|c|}
\hline & \multicolumn{2}{|c|}{ Transparent } & \multicolumn{2}{|c|}{ Opaque } & \multicolumn{2}{|c|}{ Form } \\
\hline & $\begin{array}{c}\mathrm{RT} \\
(\mathrm{msec})\end{array}$ & $\begin{array}{l}\text { Err } \\
(\%)\end{array}$ & $\begin{array}{c}\mathrm{RT} \\
(\mathrm{msec})\end{array}$ & $\begin{array}{l}\text { Err } \\
(\%)\end{array}$ & $\begin{array}{c}\mathrm{RT} \\
(\mathrm{msec})\end{array}$ & $\begin{array}{l}\text { Err } \\
(\%)\end{array}$ \\
\hline \multicolumn{7}{|l|}{ Visual Targets } \\
\hline Related & 573 & 0 & 589 & 2 & 604 & 4 \\
\hline Unrelated & 595 & 2 & 608 & 3 & 607 & 6 \\
\hline Effect & $22^{*}$ & 2 & $19^{*}$ & 1 & 3 & 2 \\
\hline \multicolumn{7}{|c|}{ Auditory Targets } \\
\hline Related & 871 & 3 & 909 & 4 & 868 & 6 \\
\hline Unrelated & 905 & 4 & 940 & 6 & 892 & 7 \\
\hline Effect & $34^{*}$ & 1 & $31^{*}$ & 2 & $24^{*}$ & 2 \\
\hline
\end{tabular}

$p<.001$, and $t(2821)=3.07, p<.01$, respectively] with no significant difference. Relative to the form condition, priming was significantly stronger for transparent items $[t(2821)=2.19, p<.05]$, but only marginally so for opaque items $[t(2821)=1.80, p=.06]$. There was no significant interaction of relatedness and priming type in the error analysis. The effects of relatedness and priming type were nevertheless significant. There was a significant overall positive priming effect $[F(1,2936)=$ $10.82, p<.05]$, as well as an increased error probability, for form items, relative to both transparent and opaque items, and for opaque items, relative to transparent items $[F(2,2936)=6.94, p<.001$; see Appendix B for an examination of this baseline difference].

Auditory targets. Only the main effects of relatedness and priming type were significant in the RT analysis. Responses were faster following related primes $[F(1,2752)=$ $46.39, p<.001]$ and for transparent and form items as compared with opaque items $[F(2,2752)=2.73, p<$ .05; see Appendix B]. The error analysis also showed a significant overall positive priming effect $[F(1,2939)=$ $6.74, p<.05]$. The effect of priming type was marginally significant $[F(2,2939)=2.68, p=.07]$, indicating an increased error probability for form items, relative to both transparent and opaque items, as well as for opaque items, relative to transparent items (see Appendix B).

\section{Discussion}

In Experiment 1, we examined masked morphological priming of stem targets with semantically transparent and opaque prefixed primes in Dutch lexical decision. The intramodal priming pattern reflects the same pattern observed with suffixed primes and stem targets in the majority of the prior research (see Rastle \& Davis, 2008, for an overview): We obtain statistically equivalent priming effects with transparent and opaque prefixed primes and no significant priming with form controls. The size of morphological priming (i.e., transparent and opaque priming minus form priming) is also similar to observations with suffixed primes (see Rastle \& Davis, 2008, Table 1). Therefore, the results of Experiment 1 are in favor of a general morpho-orthographic decomposition that operates in the same way for prefixed as for suffixed words. 
The cross-modal priming conditions revealed equivalent facilitatory priming in all three conditions. This pattern fits with prior research on intramodal auditory priming in which facilitatory priming has occurred when word or nonword primes share word-final overlap with targets (Slowiaczek \& Hamburger, 1992; Slowiaczek et al., 2000). However, when we compare this result with those of our previous study with suffixed items (Diependaele et al., 2005), we arrive at the intriguing conclusion that there is no evidence anymore for cross-modal transfer of morphological activation. Given the size of form facilitation, it can be considered that the priming due to word-final overlap is so strong that it masks any additional crossmodal facilitation. Despite this possibility, the results of Experiment 1 are mostly in line with a modality-specific (orthographic) origin of form-based morphological effects in (visual) word recognition (see, e.g., McCormick et al., 2008; Rastle \& Davis, 2008; Rastle et al., 2004).

Most importantly, Experiment 1 failed to provide evidence for effects of semantic transparency in masked morphological priming with prefixed words. ${ }^{3}$ The results nevertheless revealed two qualitative differences between transparent and opaque items that are reminiscent of the graded priming pattern in, for instance, Diependaele et al. (2005): There is a numerical advantage for transparent items, and priming only differs significantly from the form-control condition in the case of transparent items. Since observations like these reoccur across almost all related priming studies (see the overview in Rastle \& Davis, 2008), it remains possible that early influences of semantic transparency are simply more difficult to measure with the masked priming technique and the lexical decision taskperhaps because of the more abstract nature of the representations that are involved. Along this line of reasoning, the significant transparency effects with suffixed primes in the case of Diependaele et al. (2005) and other studies could be due to a stronger contrast in semantic transparency and/or a more fine-grained measurement of priming effects than is to be found in studies that have not detected such effects (including the present Experiment 1). ${ }^{4}$

The following experiments further examine fast semantic contributions to masked morphological priming with prefixed words in the light of the above considerations. Experiment 2 tested the priming conditions of Experiment 1 in reverse order - that is, we tested priming with stems as primes and prefixed words as targets. Since stems are shorter and more frequent than prefixed words, their processing should proceed faster. Hence, stem primes should activate the representations responsible for semantic transparency effects sooner and/or more strongly than prefixed primes. If a statistically significant semantic transparency effect were to emerge in Experiment 2, this would add plausibility to the idea that the qualitative differences with transparent and opaque primes in Experiment 1 reflect a real influence of semantic transparency, which potentially needs more statistical power to become significant in our analyses. We also included a reversed version of the crossmodal conditions in Experiment 2. If cross-modal priming is indeed dominated by sublexical facilitation in the pres- ence of word-final overlap, we would expect to observe a pattern similar to that seen in Experiment 1.

\section{EXPERIMENT 2}

\section{Method}

Participants. Forty undergraduate students of the University of Antwerp participated in Experiment 2. All were native speakers of Dutch and had normal or corrected-to-normal vision.

Materials and Design. For the word items, we now used the related primes of Experiment 1 as targets and the targets of Experiment 1 as related primes. We newly selected unrelated primes following the same criteria as in Experiment 1. For the nonword items, we simply reversed the order of all prime-target pairs of Experiment 1 and transformed the new targets into nonwords.

Procedure. The procedure was identical to the one used in Experiment 1.

\section{Results}

We followed the same analysis strategy as in Experiment 1 . For the RT analysis, the initial trimming led to the removal of $0.06 \%$ of the data. Table 4 gives a summary of the results.

Visual targets. The relatedness $\times$ priming type interaction was significant in the RT analysis $[F(2,2663)=5.99$, $p<.01]$ : There was a significant positive priming effect for transparent items $[t(2663)=4.60, p<.001]$ that was significantly larger than the effect for either opaque items $[t(2663)=2.73, p<.01]$ or form items $[t(2663)=3.21$, $p<.01]$. There was no significant priming (or priming differences) with either opaque or form items (see Appendix B). Relatedness $\times$ priming type was also significant in the error analysis $[F(2,2869)=4.20, p<.05]$. Further inspection revealed a trend toward a positive priming effect (i.e., smaller error probabilities following related primes) for transparent items only $(z=1.67, p=.10)$. This effect was significant relative to the form condition $(z=2.11$, $p<.05$ ), but not relative to the opaque condition.

Auditory targets. Relatedness $\times$ priming type failed to reach significance in both the RT and error analyses. In the RT analysis, there were nevertheless significant effects of relatedness and priming type, indicating an overall positive priming effect $[F(1,2642)=26.22, p<.001]$ and faster responses for form items than for transparent or opaque items $[F(2,2642)=6.98, p<.001$; see Ap-

Table 4

Condition Means and Priming Effects for Experiment 2

\begin{tabular}{|c|c|c|c|c|c|c|}
\hline & \multicolumn{2}{|c|}{ Transparent } & \multicolumn{2}{|c|}{ Opaque } & \multicolumn{2}{|c|}{ Form } \\
\hline & $\begin{array}{c}\mathrm{RT} \\
(\mathrm{msec})\end{array}$ & $\begin{array}{l}\text { Err } \\
(\%)\end{array}$ & $\begin{array}{c}\mathrm{RT} \\
(\mathrm{msec})\end{array}$ & $\begin{array}{l}\text { Err } \\
(\%)\end{array}$ & $\begin{array}{c}\mathrm{RT} \\
(\mathrm{msec})\end{array}$ & $\begin{array}{l}\text { Err } \\
(\%)\end{array}$ \\
\hline \multicolumn{7}{|l|}{ Visual Targets } \\
\hline Related & 666 & 4 & 683 & 4 & 730 & 10 \\
\hline Unrelated & 700 & 6 & 688 & 4 & 732 & 8 \\
\hline Effect & $34^{*}$ & 2 & 5 & 0 & 2 & -2 \\
\hline \multicolumn{7}{|c|}{ Auditory Targets } \\
\hline Related & 1,016 & 4 & 1,013 & 5 & 946 & 7 \\
\hline Unrelated & 1,046 & 7 & 1,044 & 6 & 971 & 8 \\
\hline Effect & $30^{*}$ & 3 & $31^{*}$ & 1 & $25^{*}$ & 1 \\
\hline
\end{tabular}

${ }^{*} p<.05$. 
pendix B]. In the error analysis, only a significant effect of relatedness emerged, indicating an overall positive priming effect $[F(1,2865)=7.02, p<.05]$.

\section{Discussion}

The intramodal data of Experiment 2 only show a significant facilitatory priming effect in the transparent condition. This effect is also significant relative to the opaque and form-control conditions. Experiment 2 thus provides clear evidence for an influence of semantic transparency. ${ }^{5}$ This outcome not only validates our semantic transparency manipulation, it also adds plausibility to the notion that the qualitative priming differences with transparent and opaque items in Experiment 1 reflect morpho-semantic activation in the early stages of processing prefixed words. The complete absence of priming for opaque items in Experiment 2 could suggest, however, that evidence for morpho-semantic and morpho-orthographic activations cannot co-occur. This can be considered as evidence against the hybrid account, ${ }^{6}$ which assumes that letter inputs are mapped in parallel onto morpho-orthographic and morpho-semantic representations (via whole-word form representations in the latter case). As noted in the introduction, the hybrid account predicts, however, that orthographic stem activation disappears for opaque words when it comes into contact with inconsistent whole-word semantic activation. This contact can be established more quickly when opaque prefixed words are preceded by stem primes than when the reverse is true, since stems activate corresponding lexical and semantic representations more rapidly than prefixed words. As such, it is possible to obtain evidence for morpho-orthographic activation with prefixed primes and stem targets, but not with stem primes and prefixed primes at a given short prime duration.

The cross-modal data replicate Experiment 1 . We again observed equivalent facilitatory priming in all three conditions. Given the clear evidence for lexically mediated effects on the intramodal trials, it would appear that - at least with short prime durations - masked cross-modal priming primarily reflects the sublexical mapping of orthography onto phonology, and that this mapping is not constrained by morphological structure. A possible explanation is that, because of the sequential nature of auditory target processing, lexical and supralexical processing emerge more slowly for such targets than for visual targets. As such, lexical and supralexical activations induced by the visual primes would require more stability to affect the processing of auditory than of visual targets. We will postpone a more detailed discussion of the cross-modal results until the General Discussion.

In the following experiments, we focused on intramodal priming. As shown by the significant effects of the control variable modality on the previous trial (see Appendix A), the mixing of auditory and visual lexical decision in Experiments 1 and 2 introduces considerable switch costs in the RTs. Although we aimed to account for this variance by introducing the control variable, residual measurement errors cannot be excluded. Given their more abstract origin, semantic transparency effects might be more vulnerable to this kind of noise (including noise from erroneous responses and switches between word and nonword responses) than are morpho-orthographic effects. ${ }^{7}$ In Experiment 3 , we therefore investigated whether we could elicit significant semantic transparency effects with the prefixed primes and stem targets of Experiment 1 when participants only had to make visual lexical decisions during the experiment. With respect to the hybrid account, it would be critical to see whether we could observe lower levels of priming in the opaque condition at the same time. Experiment 3 thus replicated Experiment 1, but the crossmodal conditions were replaced with 40 -msec intramodal priming conditions. The $40-\mathrm{msec}$ primes enabled us to study the time course of morphological activation and ensured that the same number of items was maintained per condition as in Experiment 1.

\section{EXPERIMENT 3}

\section{Method}

Participants. Forty-two undergraduate students of the University of Antwerp participated in Experiment 3. All were native speakers of Dutch and had normal or corrected-to-normal vision.

Materials and Design. The same materials were used as in the previous experiments. The only difference in the design was that the factor target modality (visual vs. auditory) was replaced by the factor prime duration ( $40 \mathrm{vs} .67 \mathrm{msec}$ ).

Procedure. The procedure was identical to that of Experiment 1, except that targets were always presented visually and primes were presented for either $40 \mathrm{msec}$ (i.e., three refresh cycles of a $75-\mathrm{Hz}$ video monitor) or $67 \mathrm{msec}$ (five refresh cycles). We thus provided the participants with instructions for a standard speeded lexical decision task. During the experiment, they encountered 72 word targets with 40 -msec primes, 72 word targets with $67-\mathrm{msec}$ primes, 72 nonword targets with $40-\mathrm{msec}$ primes, and 72 nonword targets with $67-\mathrm{msec}$ primes, all presented in random order.

\section{Results}

We followed the same analysis strategy as before. For the RT analysis, the initial trimming led to the removal of $0.09 \%$ of the data. In each analysis, we now looked at the interaction of relatedness (related vs. unrelated) with priming type (transparent vs. opaque vs. form) and prime duration ( $40 \mathrm{vs.} 67 \mathrm{msec}$ ). The set of control variables in the RT analysis now involved trial number, accuracy on the previous trial, lexicality of the previous target, and duration of the previous prime (see Appendix A). Table 5 gives a summary of the results.

In the RT analysis, the relatedness $\times$ priming type $\times$ prime duration interaction was significant $[F(2,5665)=$ $10.63, p<.001]$. With 40 -msec primes, there were no significant priming effects or priming differences across the three item types. With 67 -msec primes, there was significant facilitatory priming, but for transparent and opaque items only $[t(5665)=7.61, p<.001$, and $t(5665)=$ $4.26, p<.001$, respectively]. These effects were significant relative to the form controls $[t(5665)=6.20, p<$ .001 , and $t(5665)=3.88, p<.001$, respectively]. The priming effect for transparent items was also significantly larger than the effect with opaque items $[t(5665)=2.32$, $p<.05]$. Relatedness $\times$ priming type $\times$ prime duration was not significant in the error analysis. A significant effect occurred only for priming type $[F(2,6021)=6.31$, 
Table 5

Condition Means and Priming Effects for Experiment 3

\begin{tabular}{|c|c|c|c|c|c|c|}
\hline & \multicolumn{2}{|c|}{ Transparent } & \multicolumn{2}{|c|}{ Opaque } & \multicolumn{2}{|c|}{ Form } \\
\hline & $\begin{array}{c}\mathrm{RT} \\
(\mathrm{msec})\end{array}$ & $\begin{array}{l}\text { Err } \\
(\%)\end{array}$ & $\begin{array}{c}\mathrm{RT} \\
(\mathrm{msec})\end{array}$ & $\begin{array}{l}\text { Err } \\
(\%)\end{array}$ & $\begin{array}{c}\mathrm{RT} \\
(\mathrm{msec})\end{array}$ & $\begin{array}{l}\text { Err } \\
(\%)\end{array}$ \\
\hline \multicolumn{7}{|c|}{ 40-msec Primes } \\
\hline Related & 590 & 4 & 608 & 5 & 607 & 8 \\
\hline Unrelated & 595 & 3 & 612 & 4 & 614 & 9 \\
\hline Effect & 5 & -1 & 4 & -1 & 7 & 1 \\
\hline \multicolumn{7}{|c|}{ 67-msec Primes } \\
\hline Related & 549 & 1 & 578 & 5 & 615 & 8 \\
\hline Unrelated & 598 & 3 & 608 & 3 & 604 & 8 \\
\hline Effect & $49^{*}$ & 2 & $30^{*}$ & -2 & -11 & 0 \\
\hline
\end{tabular}

$p<.01$, reflecting an increased error probability for form items, relative to both transparent and opaque items, and for opaque items, relative to transparent items (see Appendix B).

\section{Discussion}

The results of Experiment 3 showed no priming effects with 40 -msec prime exposures but robust priming for transparent and opaque primes with 67-msec prime exposures, with significantly greater priming for the transparent items. Experiment 3 thus replicates the evidence for morpho-orthographic activations found in Experiment 1, but at the same time shows clear evidence for an influence of semantic transparency. ${ }^{8}$ We believe that the latter difference is most easily explained as the result of a more fine-grained measurement of priming effects (especially of those with a more abstract origin) when participants do not have to switch between visual and auditory lexical decision trials. More importantly, however, Experiment 3 confirms the co-occurrence of morpho-orthographic and semantic transparency effects with a short prime duration. Indeed, the emergence of a semantic transparency effect clearly did not go hand in hand with lower levels of morphological priming for opaque items (the effects even became numerically larger ${ }^{9}$ ). This is most in line with the hybrid account of morphological processing, which predicts simultaneous morpho-orthographic and morpho-semantic mappings rather than morpho-orthographic mappings that subsequently engage in semantic processing.

However, the absence of morphological effects with 40 -msec primes diverges from earlier results with suffixed words. The majority of studies have shown reliable morpho-orthographic effects (and numerical advantages for semantically transparent items) with prime durations around 40-50 msec (see Rastle \& Davis, 2008). In our own prior research (Diependaele et al., 2005), there were no effects of opaque suffixed primes and form-control primes with 40-msec exposures, as in the present case, but priming was nevertheless significant for the semantically transparent items. We have previously argued that form-based priming effects require higher prime intensities in the masked cross-modal priming paradigm because consonantal backward masks are used between primes and targets (e.g., KHRTPLZRQ; Diependaele et al., 2005;
Grainger et al., 2003). These masks can induce stronger interference with the initial form encoding of primes than is usually the case when there is no backward masking (e.g., as in Forster \& Davis, 1984). Before elaborating on the differences with earlier studies, we first look at a test of whether the presence of consonantal backward masks in Experiment 3 (used in accordance with Experiments 1 and 2) could have been responsible for the absence of morphological effects with $40-\mathrm{msec}$ prime exposures. To further enhance comparability with earlier studies, we also used the same font size for primes and targets. In the previous experiments of this study, a visual target was always presented one and a half times bigger than its prime. Clearly, this could also contribute to more extensive masking than is usually the case.

\section{EXPERIMENT 4}

\section{Method}

Participants. Forty undergraduate students at Ghent University participated in Experiment 4. All were native speakers of Dutch and had normal or corrected-to-normal vision.

Materials and Design. These were identical to the materials and design of Experiment 3.

Procedure. The procedure was identical to that of Experiment 3, except that there were no longer any backward masks (i.e., targets were presented immediately after the 40 - or $67-\mathrm{msec}$ primes) and primes and targets were both presented in 14-point Courier type.

\section{Results}

We followed the same analysis strategy as in Experiment 3. For the RT analysis, the initial trimming led to the removal of $0.6 \%$ of the data. The data for 1 participant were discarded from all analyses because of an error rate above $14 \%$. Table 6 gives a summary of the results.

The relatedness $\times$ priming type $\times$ prime duration interaction did not reach significance in the RT analysis. This was also true for the two-way interactions relatedness $\times$ prime duration and priming type $\times$ prime duration. The only interaction that was retained in the final model was relatedness $\times$ priming type $[F(2,5247)=19.48, p<.001]$. Across the two prime duration conditions, significant facilitatory priming occurred for transparent and opaque items $[t(5247)=6.12, p<.001$, and $t(5247)=2.47$, $p<.02$, respectively], and significant inhibitory priming

Table 6

Condition Means and Priming Effects for Experiment 4

\begin{tabular}{|c|c|c|c|c|c|c|}
\hline & \multicolumn{2}{|c|}{ Transparent } & \multicolumn{2}{|c|}{ Opaque } & \multicolumn{2}{|c|}{ Form } \\
\hline & $\begin{array}{c}\mathrm{RT} \\
(\mathrm{msec})\end{array}$ & $\begin{array}{l}\text { Err } \\
(\%)\end{array}$ & $\begin{array}{c}\mathrm{RT} \\
(\mathrm{msec})\end{array}$ & $\begin{array}{l}\text { Err } \\
(\%)\end{array}$ & $\begin{array}{c}\mathrm{RT} \\
(\mathrm{msec})\end{array}$ & $\begin{array}{l}\text { Err } \\
(\%) \\
\end{array}$ \\
\hline \multicolumn{7}{|c|}{ 40-msec Primes } \\
\hline Related & 616 & 3 & 656 & 5 & 681 & 9 \\
\hline Unrelated & 651 & 2 & 664 & 5 & 676 & 11 \\
\hline Effect & $35^{*}$ & -1 & 8 & 0 & -5 & 2 \\
\hline \multicolumn{7}{|c|}{ 67-msec Primes } \\
\hline Related & 618 & 2 & 647 & 5 & 699 & 7 \\
\hline Unrelated & 645 & 3 & 665 & 5 & 667 & 8 \\
\hline Effect & $27^{*}$ & 1 & $18^{*}$ & 0 & $-32^{*}$ & 1 \\
\hline
\end{tabular}


for form items $[t(5247)=2.71, p<.01]$. The facilitation with transparent items was significantly greater than the facilitation with opaque items $[t(5247)=2.58, p<.02]$. The effect with opaque items was significant relative to the form condition $[t(5247)=3.66, p<.001]$. There were no significant effects in the error analysis.

\section{Discussion}

The outcome of Experiment 4 is clear cut: The size of priming effects increased significantly from the form condition to the opaque condition and from the opaque condition to the transparent condition, for both 40- and $67-\mathrm{msec}$ primes. ${ }^{10}$ Apart from the inhibitory effect in the form-priming condition, Experiment 4 replicates the pattern observed at the longest prime duration in Experiment 3. Contrary to Experiment 3, there was no significant interaction of relatedness, priming type, and prime duration, showing that morphological priming was now also evident following $40-\mathrm{msec}$ primes. We conclude from this that the absence of priming at $40 \mathrm{msec}$ in Experiment 3 was indeed related to a procedural difference from most earlier studies: strong masking due to consonantal backward masks (and larger fonts for targets than for primes). Most importantly, Experiment 4 confirms that semantic transparency effects can be obtained with very brief prime durations. Looking more closely at the individual priming effects, it even appears that priming was not only stronger, but also arose more quickly for transparent than for opaque items. This provides a strong case for two distinct types of morphological activations during the initial stages of word recognition: morpho-orthographic and morpho-semantic.

\section{GENERAL DISCUSSION}

The present study examined masked morphological priming with prefixed words, comparing effects of (1) semantically transparent morphologically related primes (e.g., rename-name), (2) semantically opaque morphologically related primes (e.g., relate-late), and (3) formrelated primes (e.g., entail-tail). Priming effects were examined with primes and targets presented visually (intramodal priming) and with visual primes and auditory targets (cross-modal priming).

The results can be summarized as follows. With intramodal priming, both semantically transparent and opaque prefixed primes facilitated stem target recognition to a greater extent than did purely form-related items (Experiments 1,3, and 4). Furthermore, morphological priming was greater for transparent than for opaque items. This difference was robust when stems served as primes and prefixed words as targets (Experiment 2), but also when prefixed primes and stem targets appeared in a pure visual priming experiment (Experiments 3 and 4 ) - even with very brief prime exposures ( $40 \mathrm{msec}$; Experiment 4$)$. Finally, the cross-modal priming conditions showed facilitatory priming, independent of whether primes and targets were morphologically related and independent of priming direction - prefixed primes and stem targets, or vice versa (Experiments 1 and 2).

\section{Morpho-Orthographic Activation}

The fact that both semantically transparent and semantically opaque prefixed primes reveal morphological facilitation fits well with the results obtained in prior research investigating masked morphological priming with suffixed words. This outcome specifically supports the notion of a fast-acting morpho-orthographic decomposition mechanism in the processing of morphologically complex words during reading (Rastle et al., 2004). Printed complex words are assumed to become rapidly decomposed into their constituent morphemes, and this sublexical morpho-orthographic decomposition process operates on any string that can be exhaustively parsed into morphemes, even if the resulting segments do not function as morphemes in that particular string (i.e., in the case of a pseudomorphological structure). This process is thus sensitive to morphological patterns but blind to their function in the orthographic input. In this way, a prime stimulus such as relate produces activation in orthographic representations for $r e$ - and late (following the implementation of sublexical morphological decomposition proposed in the model described in Figure 1; see also Taft, 1994). This leads to increased activation in the whole-word representation of the (pseudo)stem late, thus facilitating the subsequent processing of this word when it appears as a target.

Although our study can only indirectly be compared with earlier studies on suffixed words, we believe that our results indicate that there is no critical difference in the way morpho-orthographic decomposition is accomplished for prefixed and suffixed words. The present experiments indeed show similar-sized advantages of opaque primes over form-control primes, as in earlier studies. In line with this conclusion, Duñabeitia, Perea, and Carreiras (2007) found that for suffixed and prefixed primes alike, transposing the bigram at the morpheme boundary had a detrimental effect on masked identity priming similar to the effect of inserting a new bigram (e.g., punismhent-punishment vs. punisvlent-punishment and dceode-decode vs. dviode-decode). Crucially, the same manipulation yielded significantly faster responses in the transposed condition with monomorphemic items, suggesting that the detrimental effects for derivational primes were due to disruption of the morpheme boundary. The similar effect of Duñabeitia et al.'s (2007) manipulation on suffixed and prefixed items is clearly in line with a similar process of morpho-orthographic decomposition for prefixed and suffixed words. An important consequence of this conclusion is that sublexical morphological decomposition should be characterized as a parallel mechanism (at least within the limits of visual acuity). Indeed, it appears that (at least for stems) morpho-orthographic activations are insensitive to the relative ordering of stems and affixes. As such, our results confirm previous intuitions based on the observations that (1) morpho-orthographic effects depend on the presence of an exhaustively decomposable surface structure (Longtin et al., 2003) and (2) sublexical morphological representations also play a key role in languages with 
a nonlinear morphological system, such as Hebrew (e.g., Deutsch, Frost, \& Forster, 1998).

\section{Morpho-Semantic Activation}

Our first two experiments showed a significant influence of semantic transparency when stems were used as primes and prefixed derivations as targets (Experiment 2), but not with prefixed primes and stem targets (Experiment 1). At the same time, priming for opaque items (i.e., evidence for morpho-orthographic activation) emerged with prefixed primes and stem targets (Experiment 1), but not with stem primes and prefixed targets (Experiment 2). This pattern can be explained by the idea that semantic transparency effects in priming reflect the integration of morpho-orthographic activations at the semantic level (which only succeeds for transparent derivations). This view predicts that semantic transparency effects (1) require more thorough prime processing than do effects of morpho-orthographic decomposition and (2) eliminate evidence for morpho-orthographic activation (i.e., morphological effects with opaque items; see, e.g., Longtin et al., 2003; Rastle et al., 2000). The results of Experiments 1 and 2 are in line with this, since they (1) show an effect of semantic transparency only with stem primes, which can be assumed to be processed more rapidly than prefixed primes given their shorter length and higher frequency, and (2) show no evidence for morphoorthographic activation with stem primes.

However, using only visual lexical decision trials, Experiments 3 and 4 showed that semantic transparency effects can nevertheless be obtained with prefixed primes, even with a prime duration as short as $40 \mathrm{msec}$ (Experiment 4) and in the presence of morpho-orthographic effects. Arguably the most parsimonious account of the difference with Experiment 1 is that the measurement of semantic transparency effects with derived primes and stem targets requires a certain level of precision. The analysis of Experiments 1 and 2 shows that the intermixing of visual and auditory lexical decision trials introduces switch costs (Appendix A). The variance associated with these costs cannot be fully filtered out by introducing target modality on the previous trial as a covariate. This kind of noise could especially be harmful for priming effects in the lexical decision task that arise from supralexical processing levels. Most importantly, however, the semantic transparency effects in Experiments 3 and 4 did not go hand in hand with diminished morphological priming in the opaque condition (relative to Experiment 1). This supports a double locus of morphological representations in the word recognition system, as opposed to the single locus postulated in accounts in which semantic transparency effects rely fully on morpho-orthographic decomposition. In the hybrid view, letter sequences are mapped in parallel onto sublexical (morpho-orthographic) and supralexical (morpho-semantic) representations according to their morphological structure. The latter mappings are mediated by whole-word lexical representations (Figure 1). Hence, semantic transparency effects arise independently of the type of morphological priming effects found with opaque items. This clearly accounts for the co-occurrence of both effects in the present experiments. Like the alternative views described above, the hybrid account predicts that evidence for morpho-orthographic priming gradually disappears as primes are processed more thoroughly. This follows from feedback connections spreading from supra- to sublexical levels that are absent for opaque items.

On reviewing prior masked priming results with suffixed primes and stem targets (see Rastle \& Davis, 2008), we believe that support for the hybrid view does not come as a surprise. Indeed, almost every study that has provided evidence for morphological priming with opaque items has shown a numerical advantage for transparent items (as in the present Experiment 1). Why, then, is the statistical support for fast semantic transparency effects so scarce? The present study illustrates that these effects require a more fine-grained measurement than do morpho-orthographic effects (see also Diependaele et al., 2005). This could be a general requirement for "abstract" masked priming effects in the lexical decision task. It would be very interesting to see whether semantic transparency effects are readily observed when a semantic instead of a lexical decision task is used. Possibly, considerable variation also exists in the quality and size of the semantic transparency contrasts that have been made in previous studies. In this respect, it would be interesting to know how many of the previous data sets do reveal transparency effects when continuous measures are used (and when nonlinear effects are considered as well). Clearly, future research should bring more clarity in this regard. For the time being, we believe that the consistent (factorially and continuously measured) effects of semantic transparency in our present study provide a strong case for the hybrid view of morphological processing. Furthermore, we currently see no reason to interpret statistical differences with prior studies as evidence for processing differences between prefixed and suffixed words.

\section{Cross-Modal Morphological Effects}

The results obtained in the cross-modal conditions (Experiments 1 and 2) support the modality-specific (i.e., orthographic) nature of sublexical morphological decomposition. Those conditions produced only strong facilitatory form-priming effects, which-according to findings in the auditory priming literature (Slowiaczek \& Hamburger, 1992; Slowiaczek et al., 2000)—reflect sublexical cross-modal transfer, or more specifically, fast mappings of letter codes onto phonological codes, with an emphasis on word-final (possibly rhyme) units. Contrary to the present results, in Diependaele et al. (2005) we found morphological priming for opaque suffixed primes and stem targets on both intra- and cross-modal trials. Hence, we there proposed the possibility that sublexical morphological decomposition occurs through morphologically shaped orthography-to-phonology mappings. This idea becomes less likely in the light of the present results. If anything, it would appear that such mappings are shaped according to syllabic structures. Within a bimodal architecture for word recognition (e.g., 
Diependaele, Ziegler, \& Grainger, in press; Grainger \& Ferrand, 1994), cross-modal morpho-orthographic transfer can also occur via whole-word (i.e., lexical) form representations. We believe that the present results are most in line with this alternative. The different cross-modal priming pattern with suffixed and prefixed words can be explained as follows: If a prime such as relate is presented visually, it is mapped onto morpho-orthographic representations of re-and late, which in turn activate corresponding whole-word orthographic representations. The whole-word orthographic activation for late is rapidly translated into a phonological representation. This translation (i.e., the corresponding whole-word phonological representation) is, however, inhibited by sublexical phonological representations corresponding to the prefix, which are also rapidly activated by the prime (because they mismatch in terms of initial phonemes). In the case of suffixed words, however, this translation is straightforward, since the resulting whole-word phonological activation is consistent with the word-initial phoneme activations.

Admittedly, the exact mechanisms underlying crossmodal morphological activation deserve further attention in future research. In this regard, it can be considered that, given the sequential nature of auditory target processing, masked cross-modal priming does not necessarily tap into the same prime processing stages as intramodal priming at a given prime duration. The results of Experiment 2 are particularly illustrative of this notion. They showed a clear effect of semantic transparency on intramodal trials, but only sublexical form priming on cross-modal trials. Accordingly, semantic transparency effects were only reliable on intramodal trials in our previous study with suffixed items (Diependaele et al., 2005; there were, however, numerical trends on cross-modal trials). Possibly, activations at amodal levels in the processing of visual primes require more stability (hence, higher prime intensities) to influence auditory target processing.

\section{Conclusions}

The present experiments provide support for a general type of morpho-orthographic decomposition that operates in the same way for prefixed and suffixed words. As a consequence, they are in line with previous assumptions regarding the primarily parallel nature of morphoorthographic decomposition. The present cross-modal priming results also support prior assumptions regarding the modality-specific locus of morpho-orthographic decomposition. However, the effects of semantic transparency found in the present study suggest that morphology has an additional influence on visual word recognition via (feedback from) morpho-semantic representations that are activated simultaneously with morpho-orthographic representations (via whole-word form representations). As such, the present study is mostly in line with a hybrid account of morphological processing. This account is similar to a parallel dual-route model (e.g., Schreuder \& Baayen, 1995), except that it explicitly claims that morphology exerts constraints not only on the structuring of sublexical orthographic representations, but also on the structuring of semantic representations.

\section{AUTHOR NOTE}

This research was partly supported by Grant G.0477.03 from the Fund for Scientific Research (Flanders). The authors thank Sally Andrews, Jay Rueckl, Raymond Bertram, Sarah Bernolet, and one anonymous reviewer for their helpful comments on earlier versions of the manuscript. Correspondence related to this article may be sent to K. Diependaele, Department of Experimental Psychology, Ghent University, Henri Dunantlaan 2,9000 Gent, Belgium (e-mail: kevin.diependaele@ugent.be).

\section{REFERENCES}

Baayen, R. H., Davidson, D. J., \& Bates, D. M. (2008). Mixedeffects modeling with crossed random effects for subjects and items. Journal of Memory \& Language, 59, 390-412. doi:10.1016/j .jml.2007.12.005

BaAyen, R. H., Piepenbrock, R., \& Gulikers, L. (1995). The CELEX lexical database (CD-ROM). Philadelphia: Linguistic Data Consortium, University of Pennsylvania.

BaAyen, R. H., Wurm, L. H., \& Aycock, J. (2007). Lexical dynamics for low-frequency complex words: A regression study across tasks and modalities. Mental Lexicon, 2, 419-463.

BeAuvillain, C. (1996). The integration of morphological and wholeword form information during eye fixations on prefixed and suffixed words. Journal of Memory \& Language, 35, 801-820.

Bergman, M. W., Hudson, P. T. W., \& Eling, P. A. T. M. (1988). How simple complex words can be: Morphological processing and word representations. Quarterly Journal of Experimental Psychology, 40A, 41-72.

Chateau, D., Knudsen, E. V., \& Jared, D. (2002). Masked priming of prefixes and the influence of spelling-meaning consistency. Brain \& Language, 81, 587-600.

Colé, P., Beauvillain, C., \& Segui, J. (1989). On the representation and processing of prefixed and suffixed derived words: A differential frequency effect. Journal of Memory \& Language, 28, 1-13.

Deutsch, A., Frost, R., \& Forster, K. I. (1998). Verbs and nouns are organized and accessed differently in the mental lexicon: Evidence from Hebrew. Journal of Experimental Psychology: Learning, Memory, \& Cognition, 24, 1238-1255.

DiePendaele, K., Grainger, J., \& Sandra, D. (in press). Derivational morphology and skilled reading: An empirical overview. In M. Spivey, M. Joanisse, \& K. McRae (Eds.), The Cambridge handbook of psycholinguistics. Cambridge: Cambridge University Press.

Diependaele, K., Sandra, D., \& Grainger, J. (2005). Masked crossmodal morphological priming: Unravelling morpho-orthographic and morpho-semantic influences in early word recognition. Language \& Cognitive Processes, 20, 75-114. doi:10.1080/01690960444000197

Diependaele, K., Ziegler, J., \& Grainger, J. (in press). Fast phonology and the bi-modal interactive activation model. European Journal of Experimental Psychology.

Duñabeitia, J. A., Perea, M., \& Carreiras, M. (2007). Do transposedletter similarity effects occur at a morpheme level? Evidence for morpho-orthographic decomposition. Cognition, 105, 691-703. doi:10.1016/j.cognition.2006.12.001

Duñabeitia, J. A., Perea, M., \& Carreiras, M. (2008). Does darkness lead to happiness? Masked suffix priming effects. Language \& Cognitive Processes, 23, 1002-1020. doi:10.1080/01690960802164242

Feldman, L. B., Barac-Cikoja, D., \& Kostic, A. (2002). Semantic aspects of morphological processing: Transparency effects in Serbian. Memory \& Cognition, 30, 629-636.

Forster, K. I., \& DAVIS, C. (1984). Repetition priming and frequency attenuation in lexical access. Journal of Experimental Psychology: Learning, Memory, \& Cognition, 10, 680-698.

Forster, K. I., \& Forster, J. C. (2003). DMDX: A Windows display program with millisecond accuracy. Behavior Research Methods, Instruments, \& Computers, 35, 116-124.

Giraudo, H., \& Grainger, J. (2000). Effects of prime word frequency and cumulative root frequency in masked morphological priming. Language \& Cognitive Processes, 15, 421-444. 
Giraudo, H., \& Grainger, J. (2003). On the role of derivational affixes in recognizing complex words: Evidence from masked priming. In R. H. Baayen \& R. Schreuder (Eds.), Morphological structure in language processing (pp. 209-232). Berlin: Mouton de Gruyter.

Gold, B. T., \& Rastle, K. (2007). Neural correlates of morphological decomposition during visual word recognition. Journal of Cognitive Neuroscience, 19, 1983-1993.

Grainger, J., Colé, P., \& Segui, J. (1991). Masked morphological priming in visual word recognition. Journal of Memory \& Language, 30, 370-384.

Grainger, J., Diependaele, K., Spinelli, E., Ferrand, L., \& FARIOLI, F. (2003). Masked repetition and phonological priming within and across modalities. Journal of Experimental Psychology: Learning, Memory, \& Cognition, 29, 1256-1269. doi:10.1037/0278 $-7393.29 .6 .1256$

Grainger, J., \& Ferrand, L. (1994). Phonology and orthography in visual word recognition: Effects of masked homophone primes. Journal of Memory \& Language, 33, 218-233.

HAY, J. B., \& BAAYEN, R. H. (2005). Shifting paradigms: Gradient structure in morphology. Trends in Cognitive Sciences, 9, 342-348. doi:10.1016/j.tics.2005.04.002

Hyman, L. M. (2008). Directional asymmetries in the morphology and phonology of words, with special reference to Bantu. Linguistics, 46, 309-350. doi:10.1515/LING.2008.012

Kouider, S., \& Dupoux, E. (2001). A functional disconnection between spoken and visual word recognition: Evidence from unconscious priming. Cognition, 82, B35-B49.

LAUDANnA, A., \& BurANi, C. (1995). Distributional properties of derivational affixes: Implications for processing. In L. B. Feldman (Ed.), Morphological aspects of language processing (pp. 345-364). Hillsdale, NJ: Erlbaum.

LaVric, A., Clapp, A., \& Rastle, K. (2007). ERP evidence of morphological analysis from orthography: A masked priming study. Journal of Cognitive Neuroscience, 19, 866-877.

LibBEN, G. (1994). How is morphological decomposition achieved? Language \& Cognitive Processes, 9, 369-391.

Longtin, C.-M., \& Meunier, F. (2005). Morphological decomposition in early visual word processing. Journal of Memory \& Language, 53, 26-41. doi:10.1016/j.jml.2005.02.008

Longtin, C.-M., Segui, J., \& Hallé, P. A. (2003). Morphological priming without morphological relationship. Language \& Cognitive Processes, 18, 313-334. doi:10.1080/01690960244000036

Marslen-Wilson, W., Tyler, L. K., Waksler, R., \& Older, L. (1994). Morphology and meaning in the English mental lexicon. Psychological Review, 101, 3-33.

McCormick, S. F., Rastle, K., \& Davis, M. H. (2008). Is there a "fete" in "fetish"? Effects of orthographic opacity on morpho-orthographic segmentation in visual word recognition. Journal of Memory \& Language, 58, 307-326. doi:10.1016/j.jml.2007.05.006

MEUnier, F., \& SEGUI, J. (2002). Cross-modal morphological priming in French. Brain \& Language, 81, 89-102. doi:10.1006/brln.2001.2509

Morris, J., Frank, T., Grainger, J., \& Holcomb, P. J. (2007). Semantic transparency and masked morphological priming: An ERP investigation. Psychophysiology, 44, 506-521. doi:10.1111/j.1469 -8986.2007.00538.x

Plaut, D. C., \& Gonnerman, L. M. (2000). Are non-semantic morphological effects incompatible with a distributed connectionist approach to lexical processing? Language \& Cognitive Processes, 15, 445-485.

Rastle, K., \& Davis, M. H. (2008). Morphological decomposition based on the analysis of orthography. Language \& Cognitive Processes, 23, 942-971. doi:10.1080/01690960802069730

Rastle, K., Davis, M. H., Marslen-Wilson, W. D., \& Tyler, L. K. (2000). Morphological and semantic effects in visual word recognition: A time-course study. Language \& Cognitive Processes, 15, 507-537.

Rastle, K., Davis, M. H., \& New, B. (2004). The broth in my brother's brothel: Morpho-orthographic segmentation in visual word recognition. Psychonomic Bulletin \& Review, 11, 1090-1098.

SCHREUdER, R., \& BAAYEN, R. H. (1995). Modeling morphological processing. In L. B. Feldman (Ed.), Morphological aspects of language processing (pp. 131-154). Hillsdale, NJ: Erlbaum.

SeidenberG, M. S., \& Gonnerman, L. M. (2000). Explaining deriva- tional morphology as the convergence of codes. Trends in Cognitive Sciences, 4, 353-361.

SlowiaczeK, L. M., \& Hamburger, M. (1992). Prelexical facilitation and lexical interference in auditory word recognition. Journal of Experimental Psychology: Learning, Memory, \& Cognition, 18, 1239-1250.

SlowiaczeK, L. M., McQueen, J. M., Soltano, E. G., \& Lynch, M. (2000). Phonological representations in prelexical speech processing: Evidence from form-based priming. Journal of Memory \& Language, 43, 530-560.

TAFT, M. (1994). Interactive-activation as a framework for understanding morphological processing. Language \& Cognitive Processes, 9, 271-294.

TAFT, M., \& Forster, K. I. (1975). Lexical storage and retrieval of prefixed words. Journal of Verbal Learning \& Verbal Behavior, 14, 638-647.

TAFT, M., \& Kougious, P. (2004). The processing of morphemelike units in monomorphemic words. Brain \& Language, 90, 9-16. doi:10.1016/S0093-934X(03)00415-2

WuRM, L. H. (1997). Auditory processing of prefixed English words is both continuous and decompositional. Journal of Memory \& Language, 37, 438-461.

\section{NOTES}

1. One exception is a study in Serbian by Feldman, Barac-Cikoja, and Kostic (2002). These authors found no reliable stem-priming differences between transparent and opaque prefixed primes. However, there was no strict control for form-priming effects in that study.

2. No significant priming effects occurred in the RTs or the errors for the nonword conditions. This was also the case in all of the following experiments.

3. The absence of transparency effects was confirmed in a post hoc analysis in which we looked at the interaction between our ratings of semantic transparency and relatedness in the compound RT data for transparent and opaque items (omitting the factor priming type). For both visual and auditory targets, the interaction was not significant.

4. It can be noted, in this regard, that in Diependaele et al. (2005), the critical experiment included an arguably more powerful incremental priming design.

5. In contrast to Experiment 1, the interaction between our semantic transparency ratings and relatedness now reached significance for visual targets $[F(1,1797)=8.48, p<.01]$ and accounted for all priming (i.e., after partialing it out, there was no longer a significant effect of relatedness).

6 . It can be remarked that the null effect for opaque targets could also reflect opposite priming effects with (1) targets such as recollect, which are etymologically related to the embedded stem and still convey at least some of the meaning of the stem, and (2) so-called pseudoderived targets such as recent, which are fully semantically unrelated to the embedded stem. As proposed by Taft and Kougious (2004), for instance, the semantic processing of words like recollect might still occur via abstract morphemic representations, whereas this is not the case for such words as recent. It can be predicted from this that, even though similar facilitation emerges with such pairs as recollect-collect and recent-cent, pairs like collect-recollect induce facilitation, whereas pairs like cent-recent result in inhibition. We considered this possibility in a post hoc analysis of the RT data for opaque items in Experiment 2, in which we treated items with a median semantic transparency rating above 0 as a separate partially opaque category $(N=11)$. We found no interaction of the category variable with relatedness, nor even a numerical hint of the predicted interaction (i.e., we found a 2 -msec difference between the unrelated and related conditions for partially opaque items and a $7-\mathrm{msec}$ difference for fully opaque items). For completeness, we considered the same distinction in the data of Experiment 1. In line with Longtin et al. (2003), there were again no signs of a different priming pattern.

7. This kind of noise might have been reduced in Diependaele et al. (2005), since an incremental priming design was used (including extensive training).

8. The transparency effect with $67-\mathrm{msec}$ primes was confirmed in a post hoc analysis of the RT data for transparent and opaque items. Our ratings of semantic transparency interacted with relatedness, but only in the $67-\mathrm{msec}$ condition $[t(3841)=2.21, p<.05]$. 
9. Although the priming effect in the opaque condition was numerically larger than that found in Experiment 1, only the priming difference for transparent items was significant across experiments $[t(7461)=$ $2.75, p<.01]$

10. A post hoc analysis of RTs in the transparent and opaque conditions confirmed the transparency effect by showing a significant interaction between the ratings of semantic transparency and relatedness $[t(3558)=3.26, p<.001]$. As in the main analysis, there was no threeway interaction with prime duration.

\section{SUPPLEMENTAL MATERIALS}

The word stimuli for all experiments in this article may be downloaded from http://mc.psychonomic-journals.org/content/supplemental.

\section{APPENDIXA \\ Results Regarding the Control Variables in the RT Analyses}

\section{Experiment 1}

Visual targets. Although the fixed effect of trial number was not significant, a log-likelihood ratio test showed a significant decrease in the model fit when the by-participants random term for trial number was removed $\left[\chi^{2}(1)=11.46, p<.001\right]$. There was a significant interaction of the lexical status and modality of the previous target $[F(1,2821)=21.37, p<.001]$. The RTs to the visual targets were faster following words than following nonwords if the previous target was visual [i.e., within modality; $t(2821)=4.08, p<.001$; a 26-msec difference]. Finally, there was a significant effect of accuracy on the previous trial $[F(1,2821)=26.28, p<$ $.001]$. RTs were on average $43 \mathrm{msec}$ slower following errors.

Auditory targets. Again, the fixed effect of trial number was not significant, but the by-participants random term improved the model fit significantly $\left[\chi^{2}(1)=16.01, p<.001\right]$. The interaction of the lexical status and modality of the previous target was also significant $[F(1,2752)=5.40, p<.05]$, now indicating faster responses following words if the previous target was auditory $[t(2752)=2.72, p<.01$; a 14-msec difference]. The effect of accuracy on the previous trial again indicated (slightly) slower responses following errors $[F(1,2752)=3.04$, $p<.05 ;$ a 5 -msec difference].

\section{Experiment 2}

Visual targets. The fixed effect of trial number was not significant, whereas the by-participants random term was $\left[\chi^{2}(1)=55.58, p<.001\right]$. The three-way interaction of accuracy on the previous trial, lexical status of the previous target, and modality of the previous target now reached significance $[F(1,2663)=4.43, p<.05]$. Further inspection indicated faster responses following words if the previous target was visual and had been responded to correctly $[t(2663)=6.88, p<.001$; a 46-msec difference]. Furthermore, RTs were slower following errors if the previous target had been a visually presented word $[t(2663)=2.70, p<.001$; an 86 -msec difference].

Auditory targets. The fixed effect of trial number was not significant, but the by-participants random term again improved the model fit significantly $\left[\chi^{2}(1)=28.12, p<.001\right]$. There was a significant effect of modality of the previous target $[F(1,2642)=12.91, p<.001]$, indicating slower responses following visual trials (a 14-msec difference) and a significant effect of accuracy on the previous trial $[F(1,2642)=13.61, p<.001]$, indicating slower responses following errors (a 42-msec difference).

\section{Experiment 3}

There was a significant fixed effect of trial number $[F(1,5665)=12.14, p<.01]$, as well as a significant byparticipants random term $\left[\chi^{2}(1)=56.48, p<.001\right]$. There was a general trend toward faster responses as the experiment progressed. There was also an effect of lexical status on the previous trial $[F(1,5665)=41.20, p<$ $.001]$, indicating faster responses following words (a 21 -msec difference), and an effect of accuracy on the previous trial $[F(1,5665)=67.28, p<.001]$, indicating slower responses following errors (a 26-msec difference).

\section{Experiment 4}

There was a significant fixed effect of trial number $[F(1,5247)=6.03, p<.02]$, as well as a significant byparticipants random term $\left[\chi^{2}(1)=83.24, p<.001\right]$. There was a general trend toward faster responses as the experiment progressed. There was also an effect of lexical status on the previous trial $[F(1,5247)=13.23, p<$ $.001]$, indicating faster responses following words (a $15-\mathrm{msec}$ difference), and an effect of accuracy on the previous trial $[F(1,5247)=13.85, p<.001]$, indicating slower responses following errors (a 15-msec difference). 


\section{APPENDIX B}

Baseline Analyses

In this appendix, we address a number of unanticipated baseline differences and demonstrate that they did not affect the observed priming patterns.

First of all, the error data in Experiments 1 and 3 (i.e., for stem targets) showed increased error rates for form items relative to opaque items and for opaque items relative to transparent items. Although this pattern was not evident in the baseline RTs (i.e., RTs for targets following unrelated primes), we tested whether the error proportions for our stem targets (aggregated across Experiments 1 and 3) interacted with relatedness in the RT data of Experiments 1 and 3 (excluding priming type because of its correlation with error proportion). In both experiments, there was a significant negative effect of error proportion (i.e., slower RTs for targets that induced more errors), but no interaction with relatedness (or with prime duration in Experiment 3). We therefore argue that, whatever caused the baseline difference in the error rates for stem targets, the stem-priming pattern in the RT data remained unaffected by it.

Second, the baseline RTs of Experiment 2 were significantly slower in the intramodal form condition but significantly faster in the cross-modal form condition. We reasoned that this opposite pattern could be related to the fact that (pseudo)prefixed words naturally tend to have later phonological uniqueness points (UPs) than do words of similar length without prefixes (such as the form targets in Experiment 2). In a recent study on the processing of low-frequency morphologically complex words, Baayen, Wurm, and Aycock (2007) found that whereas the UP had a (well-established) negative effect on auditory lexical decision latencies, it had a facilitatory effect on visual lexical decision latencies. They discussed the fact that, apart from auditory lexical competition, UPs also correlate with the (phonotactic) idiosyncrasy of words. More specifically, words with a late UP can be considered to be more wordlike, which provides a possible explanation for the facilitatory effect on visual lexical decision. In the present context it is important, however, to see whether we can explain the opposite baseline differences with visual and auditory targets in Experiment 2 via the UP of our derived targets and - most importantly - if so, whether this variable interacts with priming (i.e., relatedness). To this end, we separately considered four different (but correlated) measures of phonological uniqueness: (1) the plain $U P$ (taking into account all possible continuations), (2) the frequency-restricted UP (taking only continuations with a higher frequency than the target into account), (3) the (log-transformed) plain cohort frequency (i.e., the summed frequency of all word forms defining the plain UP), and (4) the frequency-restricted cohort frequency (i.e., the summed frequency of all word forms defining the frequency-restricted UP). A series of $t$ tests confirmed that all four measures yielded similar values for the targets in the transparent and opaque conditions but significantly lower values for the targets in the form condition. For the intramodal RTs in Experiment 2, the baseline difference disappeared after partialing out the effects of either the plain or the frequency-restricted cohort frequency. Neither of these measures interacted with relatedness. For the cross-modal RT data, the baseline difference only dissolved after partialing out the effect of the frequency-restricted UP. Again, there was no interaction with relatedness.

The outcome that not UP per se, but the cohort frequency explained the baseline difference for visual targets seems to align with the idea that the difference was caused by a difference in the word likelihood. The fact that, on the other hand, only the frequency-restricted UP could explain the (opposite) difference with auditory targets seems to align well with the traditional view that auditory UP effects reflect lexical competition. It is nevertheless most important for the present purposes to note that none of these variables interacted with the amount of priming, and that the priming patterns indeed remained unaltered after partialing out the effects of these variables.

Finally, there was also a significant baseline difference in the cross-modal RT data of Experiment 1, reflecting slower responses for the stem targets in the opaque condition (even after partialing out the effect of error proportion; see above). On the basis of the findings with auditory targets above, we suspected later (frequencyrestricted) UPs for the stems in the opaque condition. We reasoned that — unlike with the relatively long targets in Experiment 2-a more fine-grained localization of the frequency-restricted UP would be necessary. To this end, we first divided the auditory duration of each target (in milliseconds) by the phoneme length to obtain the average phoneme duration for each target. We then multiplied the frequency-restricted UP by this average duration to get a more precise idea of the frequency-restricted UP's location. Determined in this way, the frequencyrestricted UP did differ significantly across the three conditions, and indeed did indicate later UPs for the targets in the opaque condition. Crucially, the baseline RTs no longer differed after partialing out the effect of this UP measure. Again, the subsequent analysis (without priming type) showed no interaction with relatedness. We therefore argue that the priming pattern for auditory targets in Experiment 1 was not influenced by the presence of a baseline difference, either.

(Manuscript received August 13, 2008;

revision accepted for publication April 5, 2009.) 